\author{
Lhoussain El Fadil
}

\title{
Separability of CP-graded ring extensions
}

Received: 19 May 2010 / Accepted: 6 March 2011 / Published online: 31 July 2012

(C) The Author(s) 2012. This article is published with open access at Springerlink.com

\begin{abstract}
In this paper, we investigate separability of CP-graded ring extensions. With restrictions neither to graded fields nor to grading by torsion-free groups, we show that some results on graded field extensions given in Hwang and Wadsworth [Commun Algebra 27(2):821-840, 1999] hold.
\end{abstract}

Mathematics Subject Classification $12 \mathrm{Kxx} \cdot 15 \mathrm{~A} 63$

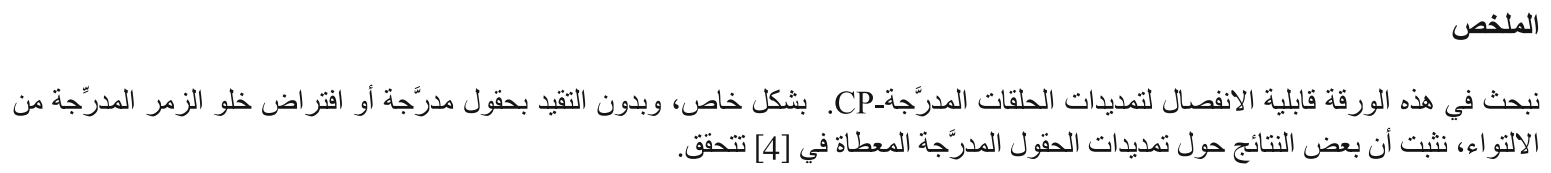

\section{Introduction}

Let $(\Gamma,+)$ be an abelian group and $R$ a unitary commutative $\Gamma$-graded ring, i.e., the ring $R$ has a decomposition $R=\oplus_{\sigma \in \Gamma} R_{\sigma}$, a direct sum of additive subgroups, such that $R_{\sigma} R_{\tau} \subset R_{\sigma+\tau}$ for every $(\sigma, \tau) \in \Gamma^{2}$. Set $R^{h}=\cup_{\sigma \in \Gamma} R_{\sigma}$ the set of homogeneous elements of $R$. For every nonzero homogeneous element $x \in R_{\sigma}$, we write $\operatorname{deg}(x)=\sigma$ and we call it the degree of $x$. If every homogeneous component of $R$ contains an invertible element, then $R$ is called a CP-graded ring. When this occurs, then $\Gamma$ is called the grade group of $R$ and for every $\sigma \in \Gamma, R_{\sigma}$ is a free $R_{0}$-module of rank 1 , which is generated by an invertible element $u_{\sigma}$. Since for every $(\sigma, \tau) \in \Gamma^{2}, \operatorname{deg}\left(u_{\sigma} u_{\tau}\right)=\sigma+\tau$, there exists a map $c: \Gamma^{2} \longrightarrow U\left(R_{0}\right)$ defined by $u_{\sigma} u_{\tau}=c_{\sigma, \tau} u_{\sigma+\tau}$, where $U\left(R_{0}\right)$ is the set of invertible elements of $R_{0}$. In that way, the CP-graded ring will be denoted by $R_{0}[\Gamma, c]$. The graded ring $R$ is said to be a graded field if every nonzero homogeneous element in $R$ is invertible. In that case, $R_{0}$ is a field and for every $\sigma \in \Gamma, R_{\sigma}$ is an $R_{0}$-vector space of dimension 1 .

In [4], Hwang and Wadsworth established some results on graded field extensions. Since their goal was to describe an algebraic extension theory of graded fields analogous to what is known for valued fields, they assumed that the grading groups are abelian torsion free. In this paper, these results are generalized to CP-graded ring extensions over a graded field.

Throughout this paper, $R=\sum_{\sigma \in \Gamma} R_{0} u_{\sigma}$ is a graded field with grading group $\Gamma$ and $S$ is a commutative graded ring, which is an extension over $R$, with grading group $\triangle$. We start showing that every homogeneous integral element of $S$ has a minimal polynomial over $R$. In Sect. 2, a characterization of separability of CPgraded ring extensions, via the discriminant ideal, is given. In particular we show that some separability results given in [4] hold for more general graded fields and grading groups. A classification theorem is given.

L. El Fadil ( $\varangle)$

Department of Mathematics, College of Sciences, King Khalid University, Abha, Saudi Arabia

E-mail: lhouelfadil@hotmail.com 


\section{Preliminaries}

The following proposition generalizes [1, Theorem 3, p. 29].

Proposition 2.1 Let $S / R$ be a CP-graded ring extension. Then $S$ is a free $R$-module such that $[S: R]=\left[S_{0}\right.$ : $\left.R_{0}\right][\triangle: \Gamma]$.

Proof For a subgroup $\Lambda$ such that $\Gamma \subseteq \Lambda \subseteq \triangle$, let $S(\Lambda):=\oplus_{x \in \Lambda} S_{x}$. In particular, $S(\Gamma)$ is a graded ring with grading group $\Gamma$ and $R \subseteq S(\Gamma) \subseteq S$. Define a new grading on $S$ over the group $\triangle / \Gamma$ by taking $S_{\sigma}:=\oplus_{x \in \sigma} S_{x}$, for every $\sigma \in \Delta / \Gamma$. Then $S$ is a $\triangle / \Gamma$-graded ring, whose homogeneous component of degree 0 is $S(\Gamma)$. Since every homogeneous component of $S$ contains an invertible element, $S$ with this $\triangle / \Gamma$-grading is a CP-graded ring such that $S(\Gamma)$ is its homogeneous component of degree 0 . In particular, $S$ is a free $S(\Gamma)$-module with a basis $\left\{w_{\sigma}, \sigma \in \Delta / \Gamma\right\}$. It follows that $S$ is a free $S(\Gamma)$-module of rank $[\triangle: \Gamma]$. On the other hand, for every $x \in \Gamma$, let $u_{x} \in R_{x}$ be an invertible element. Then $S_{x}=u_{x} . S_{0}$. As $S_{0} \otimes_{R_{0}} R=\sum_{\sigma \in \Gamma} S_{0} \otimes_{R_{0}} R_{\sigma}, S_{0} \otimes_{R_{0}} R$ is a graded ring with grading group $\Gamma$ and whose homogeneous component of degree 0 is $S_{0} \otimes_{R_{0}} R_{0}$. Hence the multiplication of $S$ induces an isomorphism of graded rings $S_{0} \otimes_{R_{0}} R \simeq S(\Gamma)$ defined by $\mu(s \otimes r)=s r$. It follows that $S(\Gamma)$ is a free $R$-module of rank $\left[S_{0}: R_{0}\right]$.

Remark that if $S$ is a finitely generated $R$-module, then $[\triangle: \Gamma]$ is called the ramification index of the extension $S / R$ and $\left[S_{0}: R_{0}\right]$ is called its residue degree.

The extension $S / R$ is called a totally ramified graded ring extension if $S$ is a finitely generated $R$-module and $[S: R]=[\triangle: \Gamma]$, i.e., $S_{0}=R_{0}$.

The extension $S / R$ is called unramified, if $S$ is a finitely generated $R$-module, $\triangle=\Gamma$ and $S_{0} / R_{0}$ is a separable ring extension.

Recall that for an $R$-algebra $S$ which is a free $R$-module of finite rank, every $x \in S$ induces an $R$-homomorphism $l_{x}$ of $S$ defined by $l_{x}(s)=x s$ for every $s \in S$. Define $T_{S / R}(x)=\operatorname{tr}\left(l_{x}\right)$ the trace of $l_{x}$. Let $M$ be a free $R$-submodule of $S$. Then $T_{S / R}$ is a linear form of $S$, which induces a bilinear form of $M$ defined by $T_{M / R}(x, y)=T_{S / R}(x y)$ for every $(x, y) \in M^{2}$. The determinant of the bilinear form $T_{M / R}$ with respect to an $R$-basis $\left(e_{1}, \ldots, e_{n}\right)$ of $M$ is denoted by $D\left(e_{1}, \ldots, e_{n}\right)$ and called the discriminant of $\left(e_{1}, \ldots, e_{n}\right)$. The discriminant ideal of the $R$-module $M$ is the principal ideal generated by $D\left(e_{1}, \ldots, e_{n}\right)$, where $\left(e_{1}, \ldots, e_{n}\right)$ is an $R$-basis of $M$. For more details see [3].

\section{Simple extensions of graded rings}

In [4], Hwang and Wadsworth have shown that if $R$ is a graded field whose grading group is a torsion free abelian group, then $R$ is an integrally closed domain. So every homogeneous integral element of $S$ over $R$ has a minimal polynomial in $R[X]$. In this section, we extend this result to the case where $\Gamma$ is an arbitrary abelian group and we give a characterization of the separability of such an element.

Let $\sigma \in \triangle$ and let $P=\sum_{i=0}^{n} a_{i} X^{i}$ be a polynomial of $R[X]$ of degree $n . P$ is said to be a $\sigma$-homogeneous polynomial if every $a_{i} \neq 0, a_{i}$ is a homogeneous element of $R$ and for every $(i, j)$ such that $a_{i} \neq 0$ and $a_{j} \neq 0, \operatorname{deg}\left(a_{i}\right)+i \sigma=\operatorname{deg}\left(a_{j}\right)+j \sigma$. Let $\lambda=\operatorname{deg}\left(a_{n}\right)+n \sigma$ be the grade of the polynomial $P$. For every $\lambda \in \Gamma+\langle\sigma\rangle$, let $R[X]_{\lambda}$ be the set of $\sigma$-homogeneous polynomials of grade $\lambda$ in $R[X]$. Then $R[X]=\sum_{\lambda \in \Gamma+<\sigma>} R[X]_{\lambda}$ is a graded ring with respect to the semigroup $\Gamma+\langle\sigma\rangle$, which will be denoted $R[X]^{(\sigma)}$. In that way $P(X)$ is a homogeneous element of degree $\lambda$ in the graded ring $R[X]^{(\sigma)}$. In particular, every polynomial of $R[X]$ splits as a sum of $\sigma$-homogeneous polynomials of $R[X]$.

Proposition 3.1 Let $\alpha$ be a homogeneous element of $S$ of degree $\sigma$. If $\alpha$ is integral over $R$, then $\alpha$ has a minimal polynomial over $R$, which is $\sigma$-homogeneous, i.e., the ideal $I(\alpha)=\{P \in R[X] \mid P(\alpha)=0\}$ of $R[X]$ is a principal ideal, which is generated by a monic $\sigma$-homogeneous polynomial.

Proof Let $x=\sum_{i=0}^{n} r_{i} \alpha^{i} \in R[\alpha]$. For every $i$, decompose $r_{i}$ as a sum of homogeneous elements of $R$. Since $\alpha$ is a homogeneous element of $S, x=\sum_{g \in \Gamma+<\sigma>} P_{g}(\alpha)$, where every $P_{g}(X)$ is a $\sigma$-homogeneous polynomial of $R[X]$ of grade $g$. Hence $R[\alpha]$ contains the homogeneous components of $x$. Consequently, $R[\alpha]$ is a graded $R$-algebra with respect to the semigroup $\Gamma+\langle\sigma>$. Since $\alpha$ is integral over $R, R[\alpha]$ is a finitely generated $R$-module. From [1, Theorem 3, p. 29], $R[\alpha]$ is a free $R$-module of finite rank. Let $P_{\alpha}(X)=\operatorname{det}\left(X I_{S}-l_{\alpha}\right)$ be the characteristic polynomial of the $R$-homomorphism $l_{\alpha}$ of $R[\alpha]$, defined by $l_{\alpha}(x)=\alpha x$. Since the degree of the polynomial $P_{\alpha}(X)$ is $[R[\alpha]: R], P_{\alpha}(X)$ is the minimal polynomial of $\alpha$ over $R$. Let $\mu_{\alpha}(X)$ 
be the $n \sigma$-homogeneous component of $P_{\alpha}(X)$ in the graded ring $R[X]^{(\sigma)}$. Then $\mu_{\alpha}(X)$ is a monic polynomial of $R[X]$ of the same degree as $P_{\alpha}(X)$. Let $\mu_{\alpha}(X)=P_{\alpha}(X) Q(X)+r(X)$ be the Euclidean division. Then degree $(r(X))<\operatorname{degree}\left(\mu_{\alpha}(X)\right)$. Since $\mu_{\alpha}(\alpha)=0$ and $P_{\alpha}(X)$ is the minimal polynomial, $r(X)=0$. Therefore, $P_{\alpha}(X)=\mu_{\alpha}(X)$ is a $\sigma$-homogeneous polynomial.

Corollary 3.2 Let $s \in S_{\sigma}$ be an invertible homogeneous element, which is integral over $R$. Then

(1) $R[s]$ is a CP-graded ring with grading group $\Gamma+\langle\sigma\rangle$.

(2) Let d be the cardinal order of $\Gamma+\langle\sigma\rangle / \Gamma, x$ a nonzero homogeneous element of $R$ of degree $d \sigma$ and $[R[s]: R]=n$. If $s$ is invertible, then $\mu_{s}(X)=x^{\frac{n}{d}} H\left(x^{-1} X^{d}\right)$ is the minimal polynomial of $s$ over $R$, where $H(X) \in R_{0}[X]$ is the minimal polynomial of $x^{-1} s^{d}$ over $R_{0}$. In particular, $R[s]_{0}=R_{0}\left[x^{-1} s^{d}\right]$.

Proof (1) Since $s$ is integral over $R$, from Proposition 2.1, $R[s]$ is a free graded $R$-algebra of finite rank. Let $\mu_{s}(X)=X^{n}+\cdots+a_{0}$ be the minimal polynomial of $s$. As $s$ is invertible and $\mu_{s}(X)$ is homogeneous, $a_{0}$ is a nonzero homogeneous element of $R$. Thus, it is invertible in $R$ and $s^{-1}=-a_{0}^{-1}\left(s^{n-1}+\cdots+a_{1}\right) \in$ $R[s]$. Therefore, $R[s]=\sum_{g \in \Gamma+<\sigma>} R_{g}$ is a CP-graded ring with grading group $\Gamma+\langle\sigma\rangle$, where $R_{g}=\sum_{\tau+n \sigma=g} R_{\tau} s^{n}$ for every $g \in \Gamma+<\sigma>$.

(2) Denote $a_{n}=1$ and let $0 \leq i<j \leq n$ such that $a_{i} \neq 0$ and $a_{j} \neq 0$. Since $\mu_{S}(X)$ is a $\sigma$-homogeneous polynomial, $(i-j) \sigma=\operatorname{deg}\left(a_{j}\right)-\operatorname{deg}\left(a_{i}\right) \in \Gamma$, and then $d$ divides $i-j$. In particular, since $a_{0} \neq 0$, if $a_{i} \neq 0$, then $d$ divides $i$. Thus, $\mu_{s}(X)=\sum_{i=0}^{m} a_{d i} X^{d i}$. Therefore, $\mu_{s}(X)=b \sum_{i=0}^{m} b^{-1} x^{i} a_{d i}\left(x^{-1} X^{d}\right)^{i}=$ $b P\left(\left(x^{-1} X^{d}\right)\right)$, where $b=x^{\frac{n}{d}}$ and $n=[R[s]: R]$. On the other hand, since $\operatorname{deg}\left(x^{-1} s^{d}\right)=-d \sigma+d \sigma=0$ and $\operatorname{deg}\left(b^{-1} x^{i} a_{d i}\right)=(i . d-n) \sigma+\operatorname{deg}\left(a_{d i}\right)=0, P(X) \in R_{0}[X]$ and $R_{0}\left[x^{-1} s^{d}\right] \subset R[s]_{0}$. By Considering the degree, $P(X)$ is the minimal polynomial of $x^{-1} s^{d}$ over $R$, and then $R[s]_{0}$ is a free $R_{0}\left[x^{-1} s^{d}\right]$-module of rank 1, i.e., $R[s]_{0}=R_{0}\left[x^{-1} s^{d}\right]$.

Remark 3.3 With notation as in the proof of Corollary 3.2, let $s \in S_{\sigma}$ be an invertible homogeneous element which is integral over $R$. Then $\mu_{s}(X)=b P\left(x^{-1} X^{d}\right)$, where $P(X)$ is the minimal polynomial of $x^{-1} s^{d}$ over $R$. In particular, if $s^{d} \in R$, then $\mu_{s}(X)=X^{d}-s^{d}$.

\section{Separable CP-graded ring extensions}

In [2], without restrictions to torsion free of grading groups, some separability results given in [4] are generalized. In this section, we investigate separability on more general CP-graded ring extensions. We close this section by a classification Theorem.

Lemma 4.1 If $S / R$ is separable, then $S$ is a free $R$-module of finite rank.

Proof From [5, Proposition III.3.2], if $S / R$ is separable, then $S$ is a finitely generated $R$-module. Thus, from Proposition 2.1, $S$ is a free $R$-module.

In the sequel of the paper, $S / R$ is a CP-graded ring extension such that $S$ is a finitely generated $R$-module and $\triangle / \Gamma=\left\{\bar{\sigma}_{1}, \ldots, \bar{\sigma}_{n}\right\}$. For every $i$, fix $w_{\sigma_{i}}$ a homogeneous element, of $S$, of degree $\sigma_{i}$. Specify $\sigma_{1}=0$ and $w_{0}=1$. Then $\left(w_{\sigma_{1}}, \ldots, w_{\sigma_{n}}\right)$ is an $S(\Gamma)$-basis of $S$.

The following theorem gives a criterion to test if a CP-graded ring extension is separable.

Theorem 4.2 Let $S / R$ be a $C P$-graded ring extension such that $S$ is a finitely generated $R$-module. Then $S / R$ is separable if and only if $S_{0} / R_{0}$ is separable and $[\triangle: \Gamma]$ is invertible in $R_{0}$.

Proof It suffices to show that $S / R$ is separable if and only if $S(\Gamma) / R$ and $S / S(\Gamma)$ are separable. Since $S(\Gamma) \simeq$ $S_{0} \otimes_{R_{0}} R, D_{R}(S(\Gamma))=D_{R_{0}}\left(S_{0}\right) R$. On the other hand, for every $(i, j)$, there exists $c_{\sigma_{i}, \sigma_{j}}$ an invertible element of $S(\Gamma)$ such that $w_{\sigma_{i}} w_{\sigma_{j}}=c_{\sigma_{i}, \sigma_{j}} w_{\sigma_{i}+\sigma_{j}}$. Thus, if $\sigma_{i}+\sigma_{j} \in \Gamma$, then the matrix of the $S(\Gamma)$-endomorphism of $S$ defined by the multiplication by $w_{\sigma_{i}+\sigma_{j}}$ is a diagonal matrix, and then $T_{S / S(\Gamma)}\left(w_{\sigma_{i}} w_{\sigma_{j}}\right)=n c_{\sigma_{i}, \sigma_{j}} w_{\sigma_{i}+\sigma_{j}}$. If $\sigma_{i}+\sigma_{j} \notin \Gamma$, then the matrix of the $S(\Gamma)$-endomorphism of $S$ defined by the multiplication by $w_{\sigma_{i}+\sigma_{j}}$ is a matrix with zero at the diagonal. Thus, $T_{S / S(\Gamma)}\left(w_{\sigma_{i}} w_{\sigma_{j}}\right)=0$. Consequently, the determinant of the bilinear form $T_{S / S(\Gamma)}$, with respect to the basis $\left(w_{\sigma_{1}} \ldots, w_{\sigma_{n}}\right)$, is $D\left(w_{\sigma_{1}} \ldots, w_{\sigma_{n}}\right)=s n^{n}$, where $s$ is an invertible element of $S(\Gamma)$. Finally, $S / R$ is separable if and only if $n$ is invertible in $R_{0}$ and $D_{R_{0}}\left(S_{0}\right) R=R$. 
Corollary 4.3 Let $S / R$ be a $C P$-graded ring extension such that $S$ is a finitely generated $R$-module.

(1) If $S / R$ is a totally ramified $C P$-graded ring extension, then $S / R$ is separable if and only if $[\triangle: \Gamma]$ is invertible in $R_{0}$. In particular, $S / S(\Gamma)$ is separable if and only if $[\triangle: \Gamma]$ is invertible in $R_{0}$.

(2) If $\Delta=\Gamma$, then $S / R$ is separable if and only if $S_{0} / R_{0}$ is separable. In particular, $S(\Gamma) / R$ is separable if and only if $S_{0} / R_{0}$ is separable.

(3) $S / R$ is separable if and only if $S(\Gamma) / R$ and $S / S(\Gamma)$ are separable.

Proposition 4.4 Let $R$ be a graded field, which is a domain with quotient field $K$ and $S / R$ a $C P$-graded ring extension such that $S$ is a finitely generated $R$-module. Then $S / R$ is separable if and only if $K S / K$ is separable.

Proof In the proof of Theorem 4.2, we have shown that $D_{R}(S)=n^{n f}\left(D_{R_{0}}\left(S_{0}\right)\right)^{n} R$, where $f=\left[S_{0}: R_{0}\right]$. Since every $R$-basis of $S$ is a $K$-basis of $K S, D_{K}(K S)=D_{R}(S) K$. Therefore $D_{K}(K S)=n^{n f}\left(D_{R_{0}}\left(S_{0}\right)\right)^{n} K$. Consequently, $K S / K$ is separable if and only if $n$ is invertible in $R$ and $D_{R_{0}}\left(S_{0}\right)=R_{0}$, i.e., $S / R$ is separable.

The following theorem gives a classification of separable CP-graded algebras over a graded field.

Theorem 4.5 Let $S / R$ be a CP-graded ring extension. Then $S / R$ is separable if and only if $S=\oplus_{i=1}^{r} S_{i}^{e_{i}}$, where $S_{i} / R$ is a separable graded field extension for each $i$. Up to an isomorphism of $R$-algebras, this decomposition is unique.

Proof Assume that $S / R$ is separable. Then $S_{0} / R_{0}$ is separable too. Hence $S_{0}=\oplus_{i=1}^{r} K_{i}$, where every $K_{i} / R_{0}$ is a separable extension of fields and let $\left(e_{i}\right)_{1 \leq i \leq r}$ be the corresponding primitive idempotents of $S_{0}(1=$ $\left.\sum_{i=1}^{r} e_{i} \in \oplus_{i=1}^{r} K_{i}\right)$. Let $S_{0}=\oplus_{i=1}^{t} K_{i}^{v_{i}}$, where $K_{i}^{v_{i}}=\oplus_{K_{j} \simeq K_{i}} K_{j}$ and let $S_{i}=\sum_{\sigma \in \triangle} K_{i} e_{i} u_{\sigma}$. Then $S_{i}$ is a graded field with grading group $\triangle$ and $S \simeq \oplus_{i=1}^{t} S_{i}^{v_{i}}$ as $R$-graded algebras.

For the uniqueness, if $f: S \longrightarrow \oplus_{i=1}^{h} T_{i}^{k_{i}}$ is an isomorphism of graded algebras. Then $f\left(S_{0}\right)$ is the homogeneous component of degree 0 of $\oplus_{i=1}^{h} T_{i}^{k_{i}}$. By Proposition 2.1, $h=r$ and for every $i, e_{i}=k_{i}$ and $T_{i} \simeq K_{i}$.

Conversely, assume that $S=\oplus_{i=1}^{r} S_{i}^{e_{i}}$, where $S_{i} / R$ is a separable graded field extension for each $i$. Then $D_{R}(S)=\prod_{i=1}^{r} D_{R}\left(S_{i}\right)=R$, i.e., $S / R$ is separable.

Acknowledgements I would like to thank Professor J. P. Tignol for his valuable comments and suggestions.

Open Access This article is distributed under the terms of the Creative Commons Attribution License which permits any use, distribution, and reproduction in any medium, provided the original author(s) and the source are credited.

\section{References}

1. Boulagouaz, M.: The graded and tame extensions. In: Lecture Notes in Pure and Applied Mathematics, vol. 153, pp. 27-40. Marcel Dekker, New York (1994)

2. El Fadil, L.: Separability of graded fields. Int. Math. Forum 1(4), 191-197 (2006)

3. El Fadil, L.; Charkani, M.: Generalization of the discriminant and applications. Arab. J. Sci. Eng. 29(1A), 93-98 (2004)

4. Hwang, Y.S.; Wadsworth, A.R.: Algebraic extensions of graded and valued fields. Commun. Algebra 27(2), 821-840 (1999)

5. Knus, M.A.; Ojanguren, M.: Théorie de la descente et algèbres d'Azumaya. In: Lecture Notes in Mathematics, vol. 389. Springer, Berlin (1974) 